\title{
Controllability of Large SEP for Earth Orbit Raising
}

\author{
Gordon Woodcock \\ Gray Research,655 Discovery Drive, Suite 300, Huntsville, AL 35806 USA, grw33@comcast.net
}

\begin{abstract}
A six-degree-of-freedom (6DOF) simulation was constructed and exercised for a large solar electric propulsion (SEP) vehicle operating in low Earth orbit. Nominal power was $\mathbf{5 0 0}$ kWe, with the large array sizes implied. Controllability issues, ineluding gravity gradient, roll maneuvering for Sun tracking, and flezible arrays, and flight control methods, were investigated. Initial findings are that a SEP vehicle of this size is controllable and could be used for orbit raising of heavy payloads.
\end{abstract}

\section{Purpose}

The research reported investigated controllability of large solar electric propulsion (SEP) systems operating in low Earth orbit. Controllability factors included gravity gradients and roll control requirements. This paper presents a status report on the work, with initial results.

\section{Introductibn}

Large solar electric propulsion systems are one option for moving large payloads from low Earth orbit (LEO) to geosynchronous orbit (GEO) or beyond ${ }^{1,2}$. Examples of such payloads are cargo elements of a logistics system for supporting human lunar exploration, and very large space optical or infrared telescopes. SEP electric power levels for these missions may range from tens to hundreds of kilowatts, with correspondingly large solar arrays. Thrust may be provided by gridded ion or Hall thrusters; in the future other thruster candidates may become competitive.

Large objects in low Earth orbit are subject to significant gravity gradient torques. Some, such as the International Space Station (ISS), are designed to fly a gravity gradient stable or neutral attitude strategy to minimize the problem. This strategy imposes on the ISS a need for two-axis solar array articulation so that the main body of the ISS remains in a local-horizontal-local-vertical (LVLH) attitude.

Another possible strategy is to control the SEP body and solar arrays to inertially face the Sun, placing thruster installations at the four corners of the (nominally square) array on two-axis gimbals. Thruster pointing controls gravity gradients and is directed to maximize available thrust for orbit raising. Restrictions on thruster pointing are applied to prevent plume impingement on the body of the vehicle. (This is a shortcoming as it will reduce the duty cycle of the thrusters and will reduce Isp and thrust due to thrust vector diversion.) Chemical propulsion provides attitude stabilization during occultation periods. This strategy was investigated several years ago during solar power satellite studies. It was concluded that multi-megawatt SEP systems could be controlled for LEO-GEO transfers by this method. The subject SEP systems were assumed constructed on orbit; it appears these configurations might be difficult for autonomous deployment.

For a SEP, it is important to minimize mass in order to minimize trip time from LEO to the destination. Given this consideration, plus a need for autonomous deployment, the attitude strategy is often single-axis solar array articulation, with roll maneuvering of the SEP body around the thrust axis, to provide Sun-pointing capability. Roll maneuvers place the solar array tilt axis perpendicular to the Sun line. Array tilt aims the array to face the Sun. This design and maneuver approach permits a conventional SEP arrangement with thrusters aft, payload forward, and - arrays extended to the sides; deployment is relatively straightforward. However, this design does not permit gravity gradient stable or neutral attitude. Thrust is available for attitude control, but thrust directed to attitude control causes at least some reduction in performance. Roll maneuvers at relatively high roll rates may be needed for precise Sun-pointing, but some loss in precision may be preferable to providing the control authority for high rates.

The SEP will at times be in Earth shadow (typically $1 / 2$ hour or less) such that electric thrust power is not available. Battery power for main propulsion operation during shadow periods represents a significant mass penalty (alpha contribution $=$ duration/[Wh $/ \mathrm{kg}]$, typically 5 to $10 \mathrm{~kg} / \mathrm{kWe}$ ). The options are to provide chemical propulsion attitude control during shadow periods, or to permit attitude drift during these periods, with recovery when main power is turned on again. 
Large solar arrays can be stiff, massive and difficult to deploy, or flexible, light and easier to deploy. There is concern about the influence of flexible arrays on SEP controllability.

In order to assess these issues, an existing six-degree-of-freedom satellite orbital simulation was modified to model the SEI problem.

\section{Mission and System Geometry; Coordinate Systems}

Requirements on the Analysis ... The SEP is in Earth orbit, and must (nominally) thrust in the direction of flight. If plane change is needed during the ascent, yaw steering (out of the orbit plane) may also be needed. The orbit must be propagated as a result of thrust, drag, and Earth oblateness effects. Gravity gradient acts on the vehicle, and its torque must be computed. The SEP needs to track the Sun, and can only generate thrust when sunlit. The location of the Sun relative to the maneuvering SEP must be determined, as well as whether or not the SEP is in Earth's shadow. The SEP configuration must permit three-axis control in order to execute roll maneuvers to track the Sun, and to maintain control of pitch and roll. The computations must be able to deal with large attitude variations, especially in roll. The SEP array tilt needed to track the Sun must be determined, and tilt control simulated. In the flexible array case, the effects of the flexible array must also be modeled.

This analysis starts with the SEP on standby in a low Earth orbit. To minimize drag and control requirements, the SEP is positioned with the solar arrays in the orbit plane and in a gravity gradient stable or neutral LVLH attitude. This is illustrated in Figure 1, which also shows some of the coordinate systems used.

Coordinate Systems ... Analysis begins in the heliocentric system, using ephemeris equations to locate the Earth according to the selected analysis start date. Since the analysis is Earth-centered, the direction of the Sun from Earth, in helio coordinates, is transformed to Earth coordinates to get the Sun vector re Earth inertial coordinates. The orbit is initialized with altitude, eccentricity, line of nodes, inclination, argument of periapsis, and iritial tnie

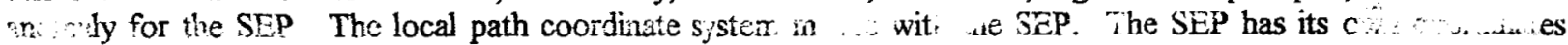
which move relative to local path coordinates according to satellite maneuvers. SEP attitude is described in terms of roll, pitch and yaw in that sequence.

The mission is transfer from low Earth orbit to geosynchronous orbit and return. A payload of 50,000 kg is delivered, and the SEP returns itself, with no payload, for reuse.

The Earth coordinate system

is $x$ towards spring equinox, $z$ north pole, y RHCS

The orbit coordinate system is the usual. $X$ points to periapsis, $Y 90$ deg in motion direction, $Z$ rhcs (north for posigrade orbit). $Z$ is the orbit vector 0 .

Satellite control strategy is roll to place array tilt axis psi, tilt to orient arrays to Sun.
The initial rate is about the $z$ axis, $y->x$ negative with attitude as shown (roll -90 ), or $x->y$ positive if $z$ axis points other way.
The local coordinate system along the path is $x$ flight direction, $y$ right facing in flight direction, $z$ nadir. The satellite initial attitude is 90 deg roll (to the left or right), with zero array tilt, to place it in gravity gradient neutral attitude

Figure 1: Coordinate Systems and SEP Axes 


\section{Estimating SEP Characteristies; SEP Representative Description}

For this analysis I chose a 500-kWe SEP in order to make the control problem potentially difficult. SEP input and calculated parameters are given in Table 1 . The SEP alpha $\vdots ;$ about $30 \mathrm{~kg} / \mathrm{kWe}$ based on d:y mass not including propeliant taniks, andi tớai tieciric power. Árray geometry and the thruster roil control oûsets were varied. Rigid arrays similar to the "square riggers" of reference 2 used a width/height ratio of 1.2 . Width is in the $y$ direction (transverse from the thrust axis) and height is along the thrust axis. A flexible deployable array used $\mathrm{w} / \mathrm{h}$ 10, which gives it a packaged length of $10+\mathrm{m}$ compatible with typical EELV payload fairing length, and an extension length of $100 \mathrm{~m}$ (each wing). Rigid analysis of this configuration was done for comparison, The flexible array was assumed to be a fan-fold assembly of rigid hinged panels $2 \times 20 \mathrm{~m}$ with $1 / 2$ meter diameter Astromast deployable trusses forward and aft of the panels. Each panel is supported at two points (one fore, one aft) by the masts. Firstmode flexible beam natural frequency was calculated based on representative mast stiffness. Total mass per unit length was masts plus panels, with result $0.0018 \mathrm{~Hz}$. For simulation development, a simpler flexible structure was also used, a rigid panel supported by a short flexible section; this had a natural frequency $0.0024 \mathrm{~Hz}$.

\begin{tabular}{|c|c|c|c|}
\hline Description ftem & Value and Units & Description ltem & Value and Units \\
\hline - Input Values - & & - Calculated Values - & \\
\hline Payload & $50000 \mathrm{~kg}$ & Up trip time & $240 d$ \\
\hline Payload length & $10 \mathrm{~m}$ & Return trip time & $61 \mathrm{~d}$ \\
\hline Power kWe & 500 & Array area & $2000 \mathrm{sq} \mathrm{m}$ \\
\hline Isp & $2000 \mathrm{sec}$ & Artay height & $10 \mathrm{~m} @ \mathrm{w} / \mathrm{h}=10$ \\
\hline Delta V up & $6700 \mathrm{~m} / \mathrm{s}$ & Array width & $100 \mathrm{~m}$ (ea wing) \\
\hline Delta V down & $6700 \mathrm{~m} / \mathrm{s}$ & lat power & $325 \mathrm{k}$ \\
\hline Irip Tine Up (Limit) & 270 days & Thrust & $33.14 \mathrm{~N}$ \\
\hline Thruster alpha & $2 \mathrm{~kg} / \mathrm{kWe}$ & Propellant flow & $0.001689 \mathrm{~kg} / \mathrm{s}$ \\
\hline PPU/cabling alpha & $4 \mathrm{~kg} / \mathrm{kWe}$ & Payload $x$ offset & $9.93 \mathrm{~m}$ \\
\hline Propulsion efficiency & $65 \%$ & Payload accomm $x$ offset & $4.93 \mathrm{~m}$ \\
\hline Run time fraction & $90 \%$ & Array $x$ offset & $-2.57 m$ \\
\hline Array W/kg & 143 & Thruster $x$ offset & $-7.5 \mathrm{~m}$ \\
\hline Array W/sq m & 250 & PPU $x$ offset & $-7.5 \mathrm{~m}$ \\
\hline Array w/h & 1.2 to 10 & Avionics/flt control $x$ offset & $-2.57 \mathrm{~m}$ \\
\hline Body diameter & $4.5 \mathrm{~m}$ & Propellant $x$ offset & $-10.07 m$ \\
\hline Initial beta tilt & O deg & Structures $x$ offset & $-5.06 m$ \\
\hline Beta tilt rate & $1 \mathrm{deg} / \mathrm{sec}$ & Payload accomm mass & $5000 \mathrm{~kg}$ \\
\hline \multirow[t]{2}{*}{ Thrust lateral offset } & \multirow{2}{*}{$\begin{array}{l}2.5 \text { or } 5 \mathrm{~m},+\&-y \\
\text { for total of } 5 \text { or } 10 \mathrm{~m}\end{array}$} & Array mass & $2500 \mathrm{~kg}$ \\
\hline & & Thruster mass & $1000 \mathrm{~kg}$ \\
\hline \multirow{2}{*}{$\begin{array}{l}\text { Avionics \& flight control } \\
\text { mass }\end{array}$} & \multirow[t]{2}{*}{$250 \mathrm{~kg}$} & PPU \& cabling mass & $2000 \mathrm{~kg}$ \\
\hline & & Propellant tank mass & $2060 \mathrm{~kg}$ \\
\hline \multirow{2}{*}{$\begin{array}{l}\text { Payload accomm. } \\
\text { fraction }\end{array}$} & \multirow[t]{2}{*}{$10 \%$ of payload mass } & Structures mass & $4483 \mathrm{~kg}$ \\
\hline & & Inert mass & $19,255 \mathrm{~kg}$ \\
\hline \multirow{2}{*}{ Propellant tank fraction } & \multirow{2}{*}{$\begin{array}{l}5 \% \text { of loaded } \\
\text { propellant }\end{array}$} & Return cutoff mass & $19,255 \mathrm{~kg}$ \\
\hline & & Up propellant & $31,394 \mathrm{~kg}$ \\
\hline \multirow[t]{2}{*}{ Structural fraction } & \multirow{2}{*}{$\begin{array}{l}35 \% \text { of other inert } \\
\text { mass }\end{array}$} & Return propellant & $7841 \mathrm{~kg}$ \\
\hline & & Unusable propellant & $1962 \mathrm{~kg}$ \\
\hline \multirow{2}{*}{$\begin{array}{l}\text { - Unused and reserve } \\
\text { propellant }\end{array}$} & \multirow{2}{*}{$\begin{array}{l}5 \% \text { of impulse } \\
\text { propellant }\end{array}$} & Total-propellant & $41,196 \mathrm{~kg}$ \\
\hline & & Total initial mass & $110,451 \mathrm{~kg}$ \\
\hline
\end{tabular}

Figure 2 illustrates the $500 \mathrm{kWe}$ SEP with the flexible deployable array. The tank is sized for xenon or krypton; with-krypton some cooling is needed to achieve desirable densities. The thrusters are on a simple deployment assembly to increase the roll control moment arm to $+/-5 \mathrm{~m}$. Rigid body control analysis showed that in the case of large $\mathrm{w} / \mathrm{h}$ (long narrow arrays) that $+/-2.5 \mathrm{~m}$ was not enough. Propulsion power processors are located close to the thrusters. Arrays and their deployment systems are forward of the tank. Avionics are included in the array area, and forward of that is the payload, assumed $5 \mathrm{~m}$ diameter by $10 \mathrm{~m}$ length. 


\section{Sinzulation Description}

Coordinate systems were described above. The simulation is run in either rigid body or flexible body mode. In the flexible case, additional inputs are reqrired to describe the flexibility of the arrays. The simulation calculites an

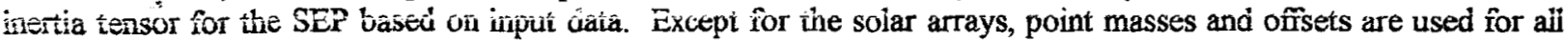
inertia contributions. In the rigid array case, array inertia contribution is computed for each wing from a flat rectangle approximation; lateral offsets are added, and a transform of the form AIA $^{-1}$ is used to account for array tilt. In the flexible array case, array inertia is not calculated because it is simulated. (Simulation includes calculation of inertia contributions.) A nominal 1-sec time step is used. Because the large SEP is not very maneuverable, this appears to provide adequate fidelity. Large pitch gimbal angles (45 degrees) are permitted so that $+/$ - pitch gimbal can exert substantial control authority.

In principle, this type of simulation is simple. Calculate the parameters needed in the appropriate coordinate system, and numerically integrate the equations of motion. There is a lot of switching back and forth between coordinate systems because changes in attitude rate are easiest to integrate in the body axis coordinate system, while the translational equations of motion and the quaternion need to be integrated in an inertial frame. Another complication is that one needs a control law to maintain correct attitude. The control law must orient the solar arrays to track the Sun and maintain body axis orientation as dictated by the desired thrust vector.

Because attitude changes can be large, SEP motion is calculated by integrating the quaternion that describes SEP attitude relative to Earth inertial coordinates. The quaternion is initialized by (1) specifying the initial date (transform from helio to Earth coordinates), the orbit parameters, the

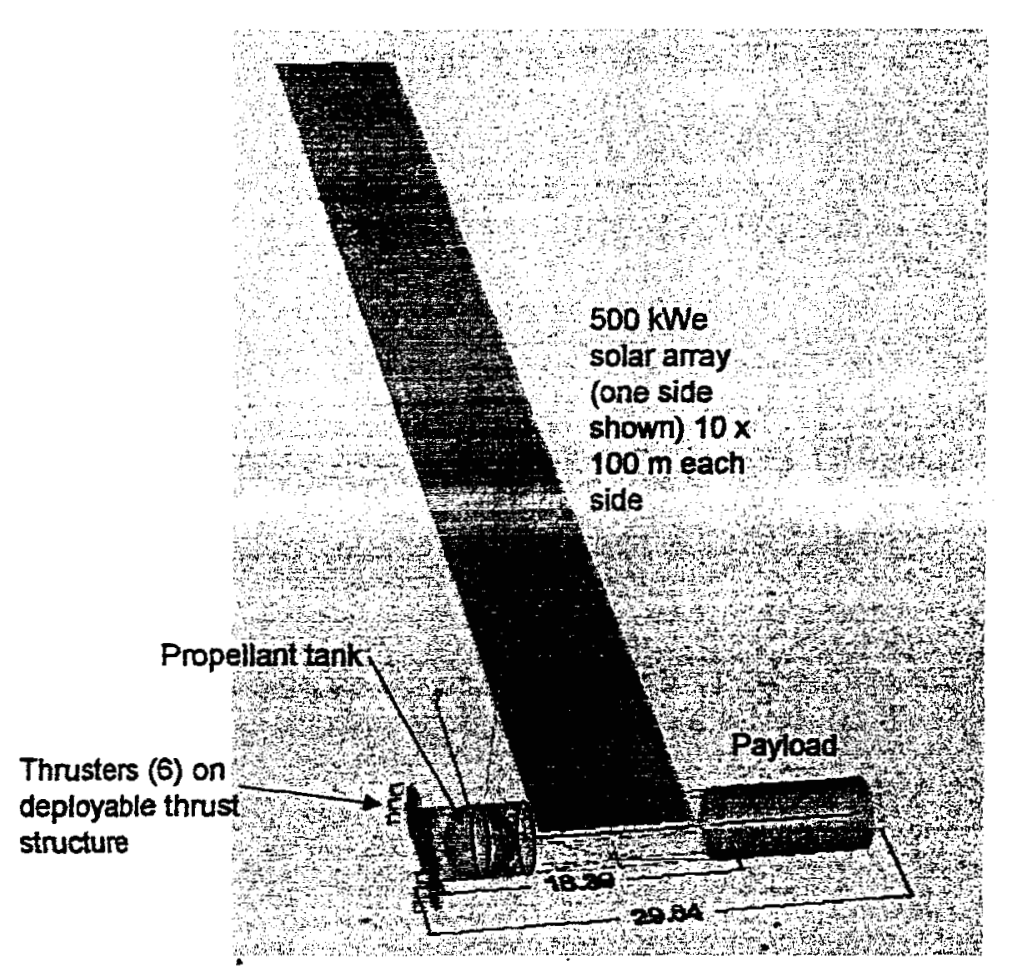

Figure 2: 500 kWe SEP, Flexible Array Geometry SEP initial true anomaly and initial attitude, (2) stepping through the coordinate transforms from Earth inertial to SEP coordinates, thus generating the transform matrix for Earth inertial to SEP coordinates, and (3) extracting the initial quaternion from the matrix.

The simulation begins as soon as the SEP is illuminated; the SEP is initially positioned in a feathered, gravity gradient stable attitude as described above. Upon illumination, thrust is switched on and the SEP begins to maneuver to full-power attitude. Array tilt and vehicle roll occur simultaneously. Array tilt is limited to a set rate, 1 degree per second in the cases presented.

\section{Rigid Results}

The first rigid tests were without power and array motion, to test the integrator. These used a SEP configuration with approximately square arrays (not the high aspect-ratio depicted above). The SEP was placed in its nominal start attitude and integrated for a few orbits. This original SEP concept was not actually gravity gradient stable in the start attitude, it was gravity gradient neutral. Because of large moments of inertia about the $y$ and $z$ axes compared to the $x$ axis, due to the distribution of large masses along the $x$ axis, the gravity gradient stable attitude is with the $x$ axis vertical. Figure 3 shows simulation test results. The first case started with the SEP in normal start attitude, and the second with an initial yaw of about 45 degrees. The first case eventually falls out of the gravity gradient neutral attitude and rotates or oscillates depending on rotating motion coupling with the slightly elliptic orbit. The second exhibits a normal gravity gradient oscillation. 
The next tests, after some debugging, simulated powered flight for about one orbit. Some adjustment of gains was necessary to obtain reasonable behavior. The thrust vector control laws were very ordinary, attitude error with negative attitude rate feedback. Array tilt used an on/off algoritbm for simulated array drive at 1 degree per second, with a $+/-1.5$ de zree deadband. At this point the roughly square array was still beirg used. Two cases are of interest: (1) during periods of occultation, no thrust is provided, and (2) during periods of occultation, chemical propulsion is used, but only for roll rate damping. These are shown in Figure 4.

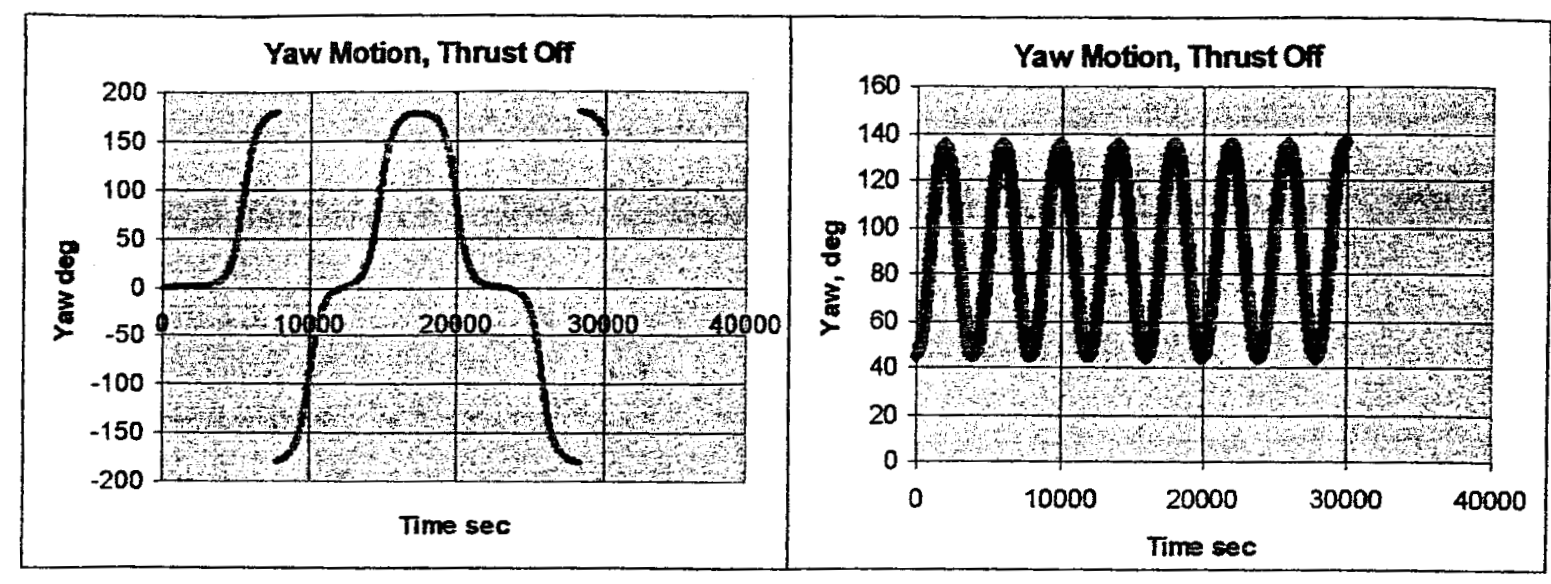

Initial yaw $=0$ (9g neutral)

Initial yaw 5 deg.ec

Figure 3: Initial Tests with No Thrust

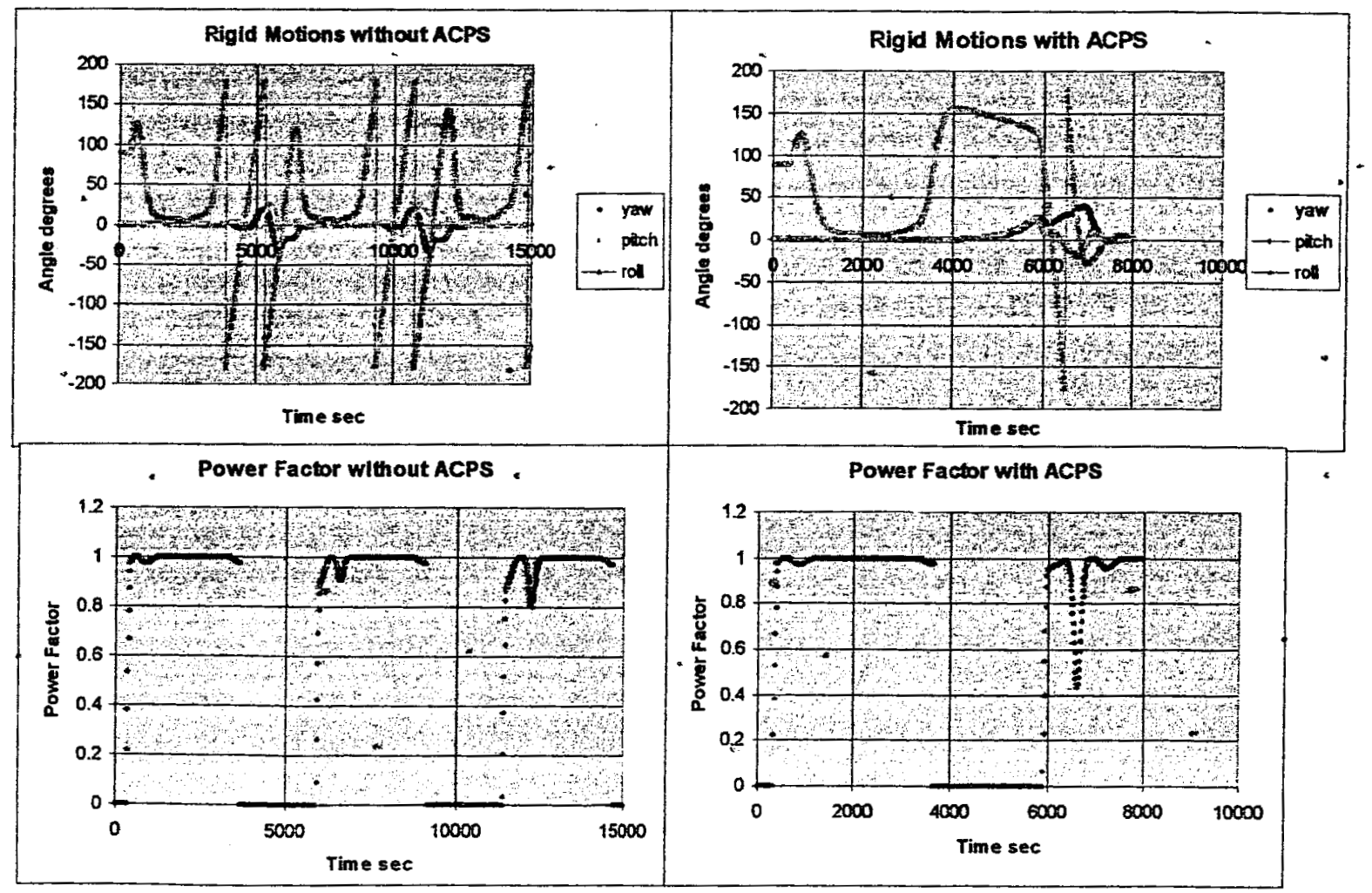

Figure 4: Effects of Attitude Control (Chemical) Propulsion on Flight Motions

American Institute of Aeronautics and Astronautics 
On the left, command roll rate increases rapidly just before the vehicle goes into occultation. High roll rate commands occur when the vehicle is flying neariy towards or nearly away from the Sun Array Sun orientation is mainly handled by array tilt, so roll errors have little effect on power factor, and power factor remains high despite considerable roll errors. However, upon entering Earth's shadow, thrust is discontinued and the high roll rate continues unaffected by commanded roll rate. The vehicle experiences approximately a complete spurious revolution. To reduce this motion, simulated chemical thrust was added. This thrust comes on whenever electric thrust is off, and is commanded only by roll rate negative feedback. The high roll rate is therefore attenuated upon entering shadow as seen on the right. Upon re-entering sunlight, the vehicle is flying nearly towards the Sun and another high roll rate occurs. There is some coupling with other axes and pitch and yaw excursions occur, but are damped out when the high roll rate is discontinued. Further adjustment of axis gains might reduce this effect. Power factor is relativelv. boed in either case. The use of chemical propulsion, even though limited, results in significant propellant consumption. Figure 5 shows a typical mass history over several orbits.

For comparison with flexible cases, further rigid runs were made with the long, narrow $\mathrm{W} / \mathrm{H}=$ 10 array. In this case, the thruster lateral offset was set at $+1-5 \mathrm{~m}$ rather than 2.5 used for the "square rigger" configuration. A 2+ day case was run to assess whether control could be retained over a large number of orbits. Orbit altitude results are shown in 'Figure 6 . Increase in eccentricity occurs because occultation is occurring during the apogee pass.

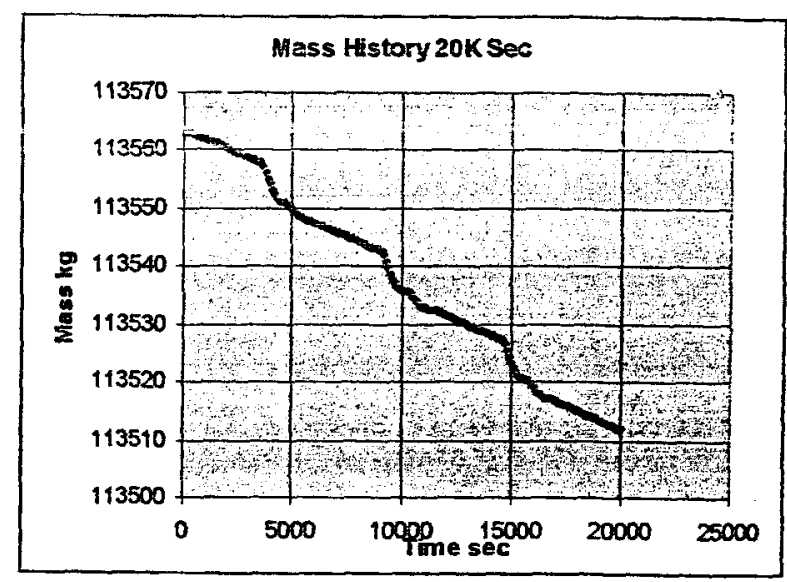

Figure 5: Mass History with ACPS

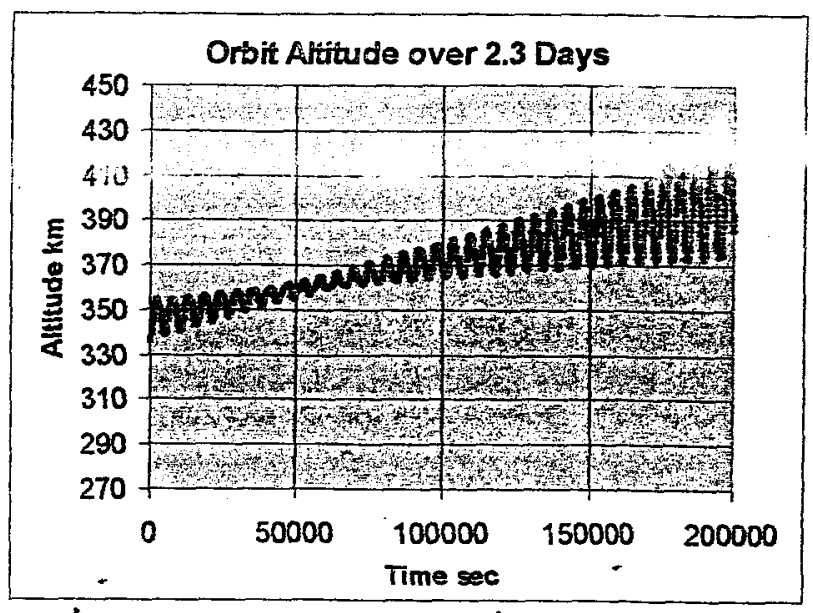

Figure 6: Orbit Altitude (Rigid Case)

\section{Flexible Array Simulation Description}

A simplified model of a flexible array was used to develop the flexible simulation and is reported here. Later, a fully flexible model will be constructed. The simplified model had a 10-meter section of flexible array as a "spring" and a 90-meter rigid section as the "mass", as illustrated in Figure 7. The flexible section had the same mast stiffness as the fully flexible array. This simplified array model had a calculated natural frequency, attached to a fixed object, of $0.0024 \mathrm{~Hz}$. This array was attached to a rigid core body. Motion of the array in the presence of core body rotation"was visualized and modeled. "Tests of the model were run to confirm the modeling before complete SEP simulations.

\section{Flexible Array Simulation Tests}

The first test did a single integration step of body roll, after which the body was fixed and the array allowed to respond. As shown in Figure 8, the numerically integrated response (after the usual debugging) exhibited the same natural frequency as analytically predicted.

Further tests were conducted to determine the response of the array to a constant applied body torque. The body begins to rotate, the flexible array lags, and torque on the rigid section of array builds up as the flexible section is bent by body motion. The array undergoes an oscillation about an overall body plus array rotation. 


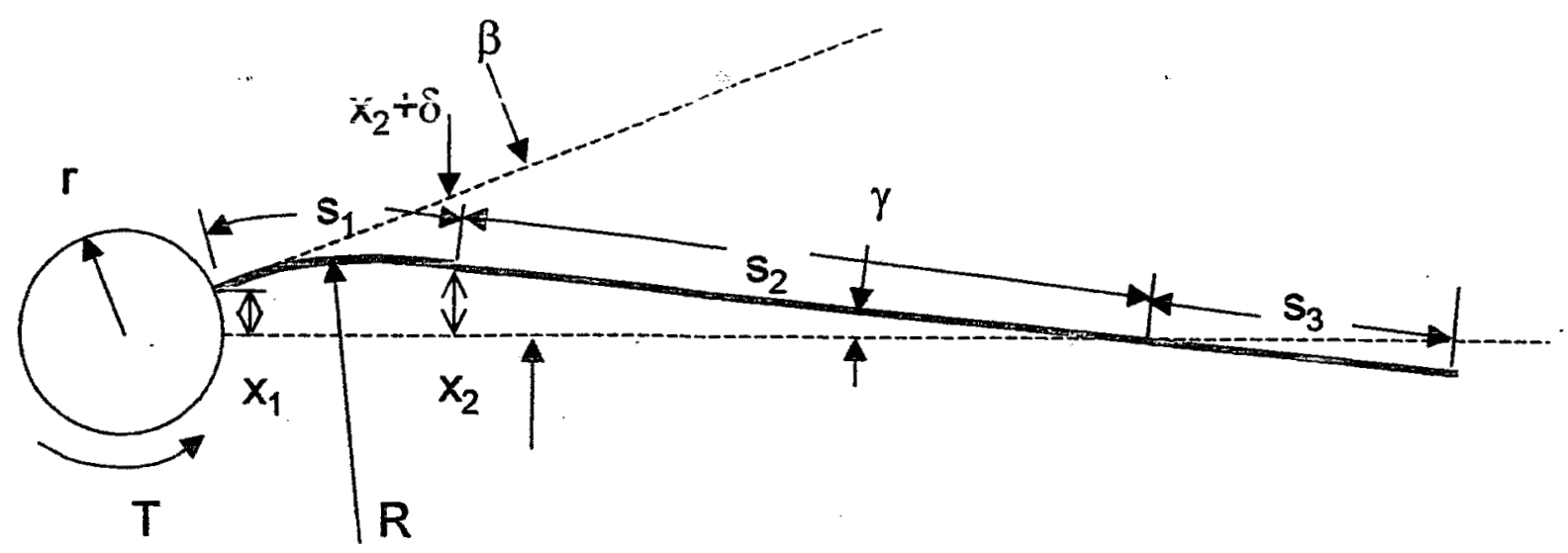

Figure 7: Diagram of Flexible Array Motion in Response to a Roll Increment. Flex Section in Blue. $S_{2}=2 S_{3}$

The frequency of this oscillation is much higher than that of the array attached to a rigid object. This was not anticipated, but should have been. The X-axis (rotational) moment of isertia of the rigid array section is about 20 times that of the rigid core body. Therefore, the oscillatory motion may be viewed as the rigid core body oscillating on the flexible spring sections of array, attached to a nearly fixed outer section of array. This motion is illustrated in Figure 9. The average roll position advances with the applied torque, and the instantaneous body roll position oscillates about the average. The oscillatory motion is greatly attenuated on the large rigid array

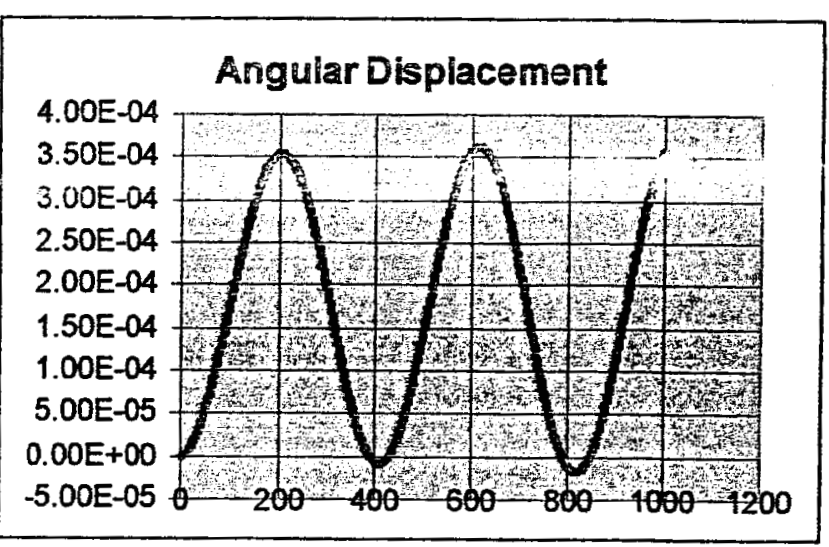

Figure 8: Oscillation Test section.

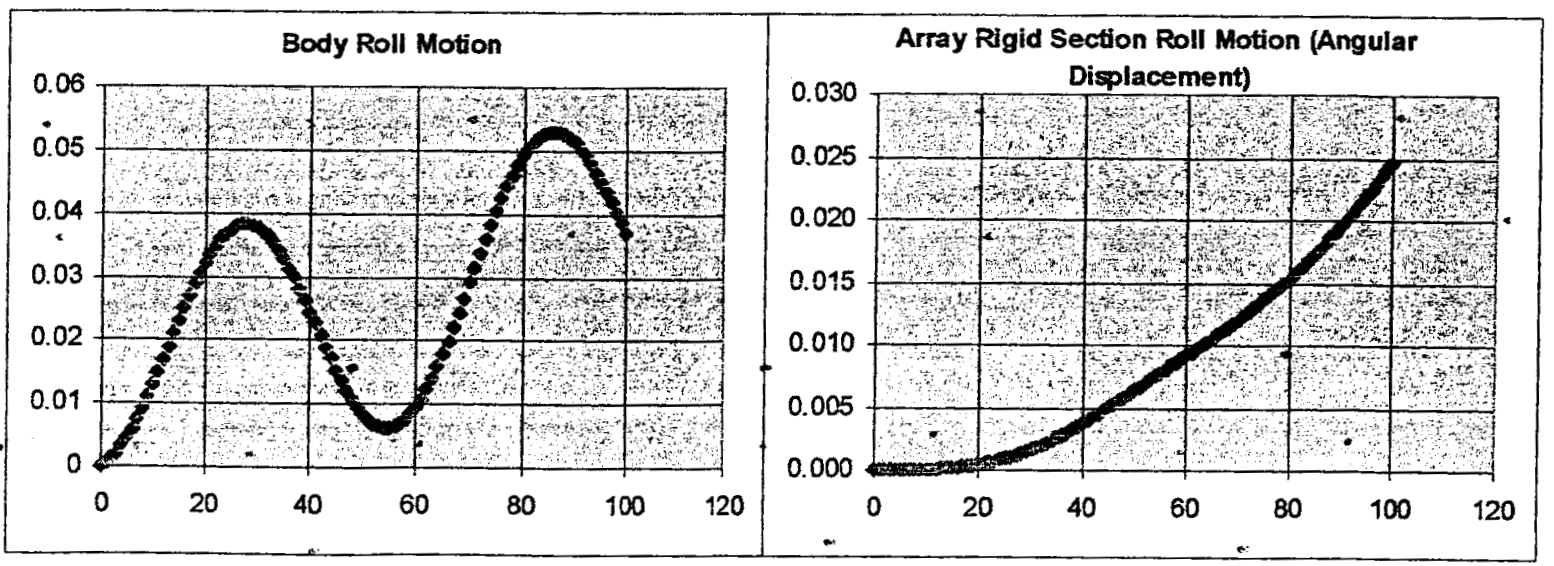

Figure 9: Test of Body and Array Roll Motion 


\section{Flexible Array SEP Control Resulls}

The simulated flexible array was then connected to the 6DOF simulation to analyze controllability of the large SEP. In addition to the simplification of the array flexibility, a furt er simplification was added in that the flexible

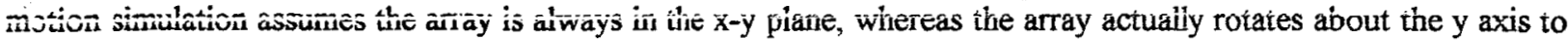
track the Sun. This means that the flexible response is always maximum, since roll motion is about the $\mathrm{x}$ axis. Pitch and yaw motions in this current simulation are small compared to roll motions.

In the case of orbit raising with plane change, yaw motion can be more significant, since yaw steering is required to change the plane. The yaw motion in that case is like a sinusoidal oscillation in yaw attitude with one oscillation per orbit.

Figure 10 shows motions for the rigid case and the flexible case, for the same flight conditions. Figure 11 shows the motion of the rigid body and the flexible array; note that the array lags as would be expected but not greatly. Oscillations are not present and are apparently damped out by the negative rate feedback in the control law.

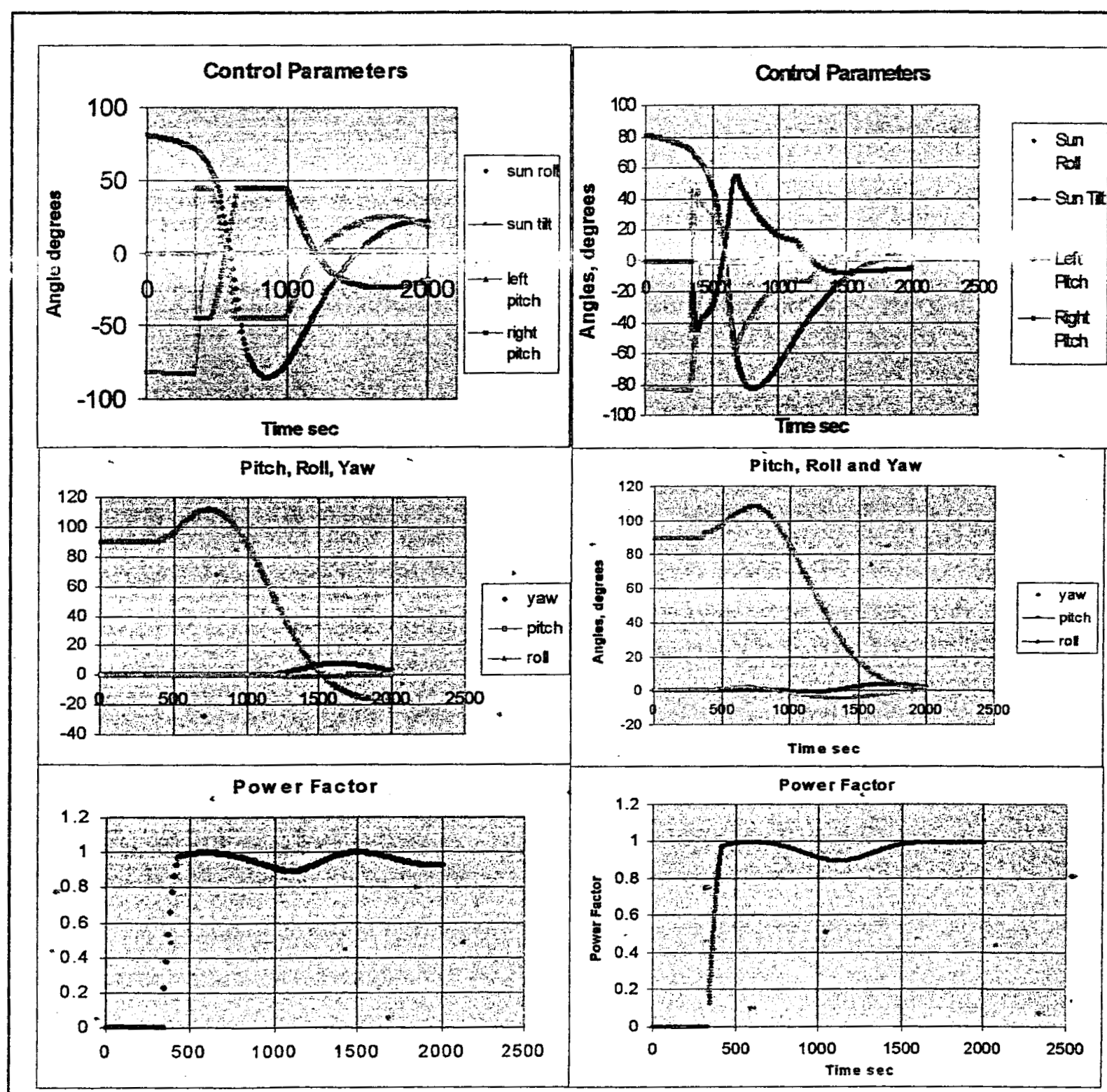

Figure 10: Rigid (Lefi) and Flexible (Right) Flight Motions Compared 


\section{Findings to Date}

Results of this study at this point are tentiatitye; it is a wotk in progress. The riain results are three:

(1) Large SEP vehicles are controllable in low Earth orbit, at least enough so to perform orbit raising. The main control problem is roll maneuvers of large vehicles.

(2) Use of some chemical attitude control propulsion during occultation periods may be needed. Our results were somewhat inconclusive and need more investigation. If needed, the performance

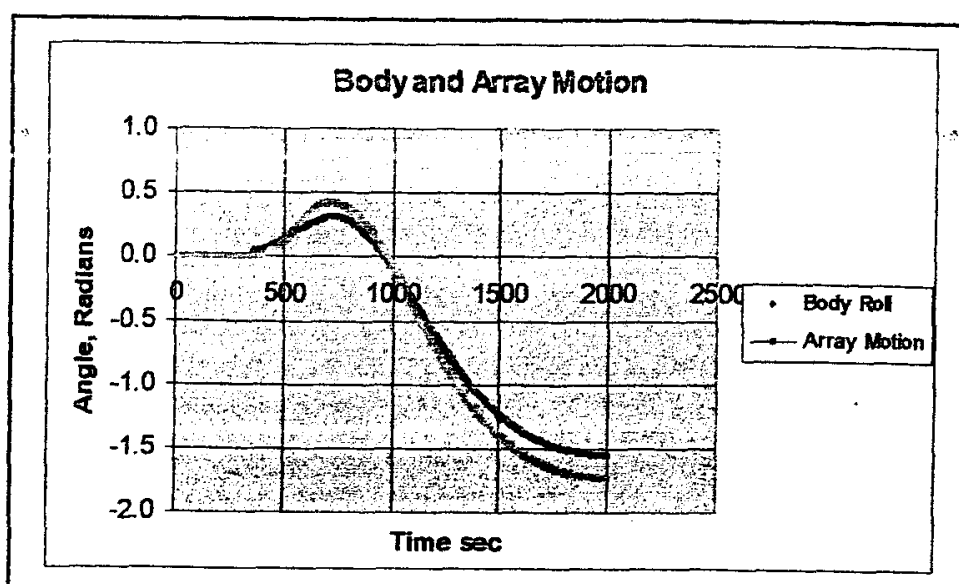

Figure 11 Body and Flexible ArTay Motion impact will be significant, due to propellant consumption at low Isp. See below regarding an anticipatory control scheme that could reduce or eliminate this problem. Also note that all control problems are ameliorated with altitude, since the orbit period is longer and maneuver rates are reduced.

(3) Large flexible arrays, at least up to about $500 \mathrm{kWe}$, even with very low natural frequencies, are controllable. The actuators (thrusters) and attitude sensors are on a central relatively rigid body, and flexible motions of large anrays appear as an external disturbance, which is sontrolled by negative rate feedback. $i$; ivine rincisiers are feeble actuators, and even with very flexible arrays, do not introduce array attitude errors more than a few degrees. Electric propulsion systems do not require precise attitude control. Trajectory errors introduced by thrust vector errors can be compensated by GN\&C. Array solar-pointing attitude is only a power function, and since the power loss is a cosine of the attitude error, errors of a few degrees are negligible.

\section{Issues and Future Work}

Gain Tuning - Very little gain tuning was performed. Further effort could improve controllability and performance. Gains on the 3 axes were the same; variations here might prove fruitful.

- Fully Flexible Array: Array Configuration - The flexible array model was crude. A fully flexible array needs to be modeled and simulated. Only two array configurations were tried. Concept design studies relating ease of array deployment and array mass versus configuration and stiffness should be performed, with simulation of the most promising concepts to ensure that they are controllable and provide adequate overall flight performance.

Correct Modeling of Flexible Array Attitude - The flexible array was modeled as always in the $x^{-}-y$ plane. This appears to be a worst case. It is not, of course, accurate, since the array is tilted to face the Sun. A higher fidelity model needs to be constructed and exercised to account for actual array attitude versus time, its actual flexible response to body torques applied by the thrusters, and overall influence on flight control and performance.

Anticipatory Controller - The typical proportional-differential controller simulated here is designed to respond reactively to unpredictable disturbances. Command roll-attitude in an orbit is, of course, highly predictable. In this situation of a large vehicle hard to maneuver, a better control system would anticipate required maneuvers, especially .where high rates are demanded, and initiate -response to the anticipated requirement before the requirement appears as an attitude error. Maximum rates and overshoots could be reduced, and better overall attitude performance achieved.

Yaw Steering - Orbit raising with plane change requires yaw steering to accomplish the plane change. This complicates the attitude control problem by adding another degree of freedom to required maneuvers. (The simulation already has all the degrees of freedom, but attitude commands, except to control pitch and yaw at zero, appear only for the roll axis.) Orbit raising with plane change needs to be simulated to determine whether the 
additional degree of freedom on commands leads to attitude rate coupling on the various axes. Some of this is observed even in the present simulation.

Performance - Orbit raising nerformance was explored very little There are potentially significant loss mechanisms, including off-pointing of the Sun and the thrust vector, and losses due to chemical propulsion used to control attitude during occultation periods. These need to be explored to obtain estimating relations and rules of thumb for overall performance prediction for large SEP vehicles, so that realistic performance analysis can be performed without necessity for detailed simulations.

Exploration of the Analysis Space - Finally, the large and complex analysis space (all the parameters, mission requirements, flight conditions, configurations, etc.) was only glimpsed. First, a reasonable plan for exploration of the analysis space needs to be created in order to do the important exploration as economically as practicable, and to neglect areas of exploration without significant impact. The plan needs to be executed to the level needed to obtain design guidelines for SEP mission analysis and design. When a specific system is designed and built, detailed simulation will be performed in depth for that system. What is needed now is enough work to facilitate performance estimates for conceptual and preliminary design, with reasonable confidence in results.

\section{References}

1. Gefert, Leon P., Hack, Hurt J., \& Kerslake, Thomas W., "Options for the Human Exploration of Mars using Solar Electric Propulsion", CP458, Space Technology and Applications International Forum - 1999, The American Institute of Physics.

2. Sarver-Verhey, Timothy R., Kerslake, Thomas W., Rawlin, Vincent K., Falck, Robert, Dudzinski, Leonard J., and Oleson, Steven R., Solar Electric Propulsion Vehicle Design Study for Cargo Transfer to Earth-Moon L1", AIAA paper 2002-3791, Joint Propulsion Conference 2002. 